\title{
Asymptotic stability of standing waves for the coupled nonlinear Schrödinger system
}

\author{
Yang Liao ${ }^{1 *}$, Quanbao Sun ${ }^{2}$, Xin Zhao ${ }^{3}$ and Ming Cheng ${ }^{4}$
}

\section{"Correspondence:}

yangliaomath@163.com

'school of Mathematics and

Information Science, Henan

University of Economics and Law,

Zhengzhou, 450000, P.R. China

Full list of author information is

available at the end of the article

\begin{abstract}
This paper considers the asymptotic stability of standing waves of a coupled nonlinear Schrödinger system with an attractive potential. Meanwhile, the existence of a center manifold is obtained.
\end{abstract}

Keywords: asymptotic stability; center manifold; Schrödinger equation

\section{Introduction and main results}

The two-component system of time-dependent nonlinear Schrödinger equations arises in the binary mixture of Bose-Einstein condensates with two different hyperfine states (see [1]):

$$
\begin{aligned}
& i \hbar \partial_{t} u+\frac{\hbar^{2}}{2 m} \Delta u-V_{1}(x) u-a_{1}|u|^{2} u-a_{2}|v|^{2} u=0, \\
& i \hbar \partial_{t} v+\frac{\hbar^{2}}{2 m} \Delta v-V_{2}(x) v-a_{3}|v|^{2} v-a_{4}|u|^{2} v=0,
\end{aligned}
$$

where $u(x, t)$ and $v(x, t)$ denote the wave functions, $\hbar$ is the Planck constant divided by $2 \pi$, $m$ is atom mass, $V_{i}$ is the trapping potential for the $i$ th hyperfine state, $a_{i} \geq 0, i=1,2,3,4$, is the associated axial frequency.

By rescaling and some simple assumptions, the above system could be viewed as the coupled nonlinear Schrödinger system:

$$
\begin{aligned}
& i \partial_{t} u+\Delta u-V_{1}(x) u-a_{1}|u|^{2} u-a_{2}|v|^{2} u=0, \\
& i \partial_{t} v+\Delta v-V_{2}(x) v-a_{3}|v|^{2} v-a_{4}|u|^{2} v=0, \quad x \in \mathbb{R}^{2}, t>0,
\end{aligned}
$$

with initial data

$$
u(0, x)=u_{0}(x), \quad v(0, x)=v_{0}(x) .
$$

In the present paper, we assume that $a_{2}=a_{4}$.

In the last decades, there has been a lot of interest in the study of localized modes in the coupled nonlinear Schrödinger system. In particular, for the existence of standing wave (periodic in time and exponentially localized in space) for various coupled nonlinear

\section{然 Springer}

(c) 2014 Liao et al.; licensee Springer. This is an Open Access article distributed under the terms of the Creative Commons Attribution License (http://creativecommons.org/licenses/by/4.0), which permits unrestricted use, distribution, and reproduction in any medium, provided the original work is properly credited. 
Schrödinger system, one may refer to [2-6] for more details. As we know, there are few results on the asymptotic stability of standing waves for the coupled nonlinear Schrödinger system (1.1)-(1.2).

Assume that the standing wave of system (1.1)-(1.2) has the form as follows:

$$
u_{E_{1}}(t, x)=e^{-i E_{1} t} \psi_{E_{1}}(x), \quad v_{E_{2}}(t, x)=e^{-i E_{2} t} \phi_{E_{2}}(x),
$$

where $E_{1}, E_{2} \in \mathbb{R}$ and $\psi_{E_{1}}, \phi_{E_{2}} \in \mathbf{H}^{2}\left(\mathbb{R}^{2}\right)$ satisfy the time independent equations:

$$
\begin{aligned}
& {\left[-\triangle+V_{1}\right] \psi_{E_{1}}+a_{1}\left|\psi_{E_{1}}\right|^{2} \psi_{E_{1}}+a_{2}\left|\phi_{E_{2}}\right|^{2} \psi_{E_{2}}=E_{1} \psi_{E_{1}},} \\
& {\left[-\Delta+V_{2}\right] \phi_{E_{2}}+a_{3}\left|\phi_{E_{2}}\right|^{2} \phi_{E_{2}}+a_{4}\left|\psi_{E_{1}}\right|^{2} \phi_{E_{2}}=E_{2} \phi_{E_{2}} .}
\end{aligned}
$$

Here, we give some notations. Assume that $\langle x\rangle=\left(1+|x|^{2}\right)^{\frac{1}{2}}$. For $\sigma \in \mathbb{R}$, the Sobolev space $L_{\sigma}^{2}$ with the norm $\|f(x)\|_{L_{\sigma}^{2}}=\left\|\langle x\rangle^{\sigma} f(x)\right\|_{L^{2}}$ denotes the space of functions $f(x)$ such that $\langle x\rangle^{\sigma} f(x)$ are square integrable. $C_{a, b, \ldots}$ denotes a constant depending on $a, b, \ldots$

Define

$$
\begin{aligned}
& X=(\psi, \phi)^{T}, \\
& F\left(\psi_{E_{1}}, \phi_{E_{2}}\right)=\left(a_{1}\left|\psi_{E_{1}}\right|^{2} \psi_{E_{1}}+a_{2}\left|\phi_{E_{2}}\right|^{2} \psi_{E_{2}}, a_{3}\left|\phi_{E_{2}}\right|^{2} \phi_{E_{2}}+a_{4}\left|\psi_{E_{1}}\right|^{2} \phi_{E_{2}}\right)^{T}, \\
& \mathcal{A}=\left(\begin{array}{cc}
-\triangle+V_{1} & 0 \\
0 & -\triangle+V_{2}
\end{array}\right),
\end{aligned}
$$

and

$$
\mathcal{E}=\left(\begin{array}{cc}
E_{1} & 0 \\
0 & E_{2}
\end{array}\right)
$$

Then the system (1.3)-(1.4) can be rewritten as

$$
\mathcal{A} X-\mathcal{E} X=F
$$

It is obvious that $X \equiv 0$ is a trivial solution of (1.5). In order to make a bifurcation with a nontrivial, one parameter family of solutions, we need (iii) in the following assumptions:

(A) Assume that:

(i) There exist $C$ and $\alpha$ such that

$$
\left|V_{i}(x)\right| \leq C\langle x\rangle^{-\alpha}, \quad i=1,2, \forall x \in \mathbb{R}^{2} .
$$

Note that potential $V_{1}, V_{2}$ could be different in our paper.

(ii) 0 is a regular point of the spectrum of the linear operator $-\Delta+V_{i}$ acting on $\mathbf{L}^{2}$, $i=1,2$.

(iii) For $i=1,2,-\triangle+V_{i}$ acting on $\mathbf{L}^{2}$ has exactly two negative eigenvalues $\omega_{i}$ with corresponding normalized eigenvectors $\psi_{0}$ and $\phi_{0}$, respectively. It is well known that $\psi_{0}$ and $\phi_{0}$ are exponentially decaying as $|x| \rightarrow \infty$, and they could be chosen strictly positive. 
The main result of this paper is the following.

Theorem 1.1 Assume that hypothesis (A) holds. Then there exists an $\varepsilon_{0}>0$ such that for any fixed $\sigma>1$ and the initial data $u_{0}, v_{0}$ satisfying

$$
\begin{aligned}
& \max \left\{\left\|u_{0}\right\|_{\mathbf{L}_{\sigma}^{2}},\left\|u_{0}\right\|_{\mathbf{H}^{1}}\right\} \leq \varepsilon_{0}, \\
& \max \left\{\left\|v_{0}\right\|_{\mathbf{L}_{\sigma}^{2}},\left\|v_{0}\right\|_{\mathbf{H}^{1}}\right\} \leq \varepsilon_{0},
\end{aligned}
$$

the initial value problem (1.1)-(1.2) is globally well-posed in $\mathbf{H}^{1} \times \mathbf{H}^{1}$.

Moreover, the solution of the initial value problem (1.1)-(1.2) has the form

$$
\begin{aligned}
& u(t, x)=a(t) \psi_{0}(x)+h_{1}(a(t))+r_{1}(t, x), \\
& v(t, x)=b(t) \phi_{0}(x)+h_{2}(b(t))+r_{2}(t, x),
\end{aligned}
$$

with

$$
\begin{aligned}
& \left\|r_{i}(t)\right\|_{\mathbf{L}_{-\sigma}^{2}} \leq C_{p_{0}} \varepsilon_{0}(1+|t|)^{2 p_{0}^{-1}-1}, \\
& \left\|r_{i}(t)\right\|_{\mathbf{L}^{p}} \leq C_{p, p_{0}} \varepsilon_{0}(1+|t|)^{2 p_{0}^{-1}-1} \log ^{\frac{1-2 p_{0}^{-1}}{1-2 p_{0}^{-1}}}(2+|t|), \quad i=1,2,
\end{aligned}
$$

where $2 \leq p \leq p_{0}$.

This paper is organized as follows. In the next section, we show the existence of a center manifold of the coupled nonlinear Schrödinger system (1.1)-(1.2). We investigate the asymptotic stability of the system (1.1)-(1.2) in Section 3.

\section{The center manifold}

This section is devoted to the proof of the existence of a center manifold. The method of constructing the center manifold is based on the standard bifurcation argument in Banach spaces for (1.3)-(1.4) at $\left(\omega_{1}, \omega_{2}\right)^{T}$ (see [7]). Since the spectrum of the operator $-\Delta+V_{i}$ has a discrete and continuous part, we follow the idea of $[8,9]$ and decompose the solution of (1.5) in its projection onto the discrete and continuous part:

$$
\begin{aligned}
& \psi_{E_{1}}=a \psi_{0}+h_{1}, \quad a=\left\langle\psi_{0}, \psi_{E_{1}}\right\rangle, \quad h_{1}=P_{c} \psi_{E_{1}}, \\
& \phi_{E_{1}}=b \phi_{0}+h_{2}, \quad b=\left\langle\phi_{0}, \phi_{E_{2}}\right\rangle, \quad h_{2}=P_{c} \phi_{E_{2}} \text {, }
\end{aligned}
$$

where $P_{c}$ denotes the projector onto the continuous spectrum of $-\triangle+V_{i}$ in $L^{2}$.

Now, projecting (1.5) onto $X_{0}=\left(\psi_{0}, \phi_{0}\right)^{T}$ and its orthogonal complement, i.e. range $P_{c}$, we have

$$
\begin{aligned}
& X=(\mathcal{A}-\mathcal{E})^{-1} P_{c} F_{1}(a, b, X), \\
& \mathcal{E}_{0}-\mathcal{E}=-\mathcal{B}^{-1} F_{2}(a, b, X),
\end{aligned}
$$

where

$$
X=\left(\begin{array}{l}
h_{1} \\
h_{2}
\end{array}\right), \quad \mathcal{B}=\left(\begin{array}{ll}
a & 0 \\
0 & b
\end{array}\right), \quad \mathcal{E}_{0}=\left(\begin{array}{cc}
\omega_{1} & 0 \\
0 & \omega_{2}
\end{array}\right), \quad \mathcal{E}=\left(\begin{array}{cc}
E_{1} & 0 \\
0 & E_{2}
\end{array}\right),
$$


and

$$
\begin{aligned}
& F_{1}(a, b, X)=F\left(a \psi_{0}+h_{1}, b \phi_{0}+h_{2}\right), \\
& F_{2}(a, b, X)=\left(\left\langle\psi_{0}, a_{1}\left|a \psi_{0}+h_{1}\right|^{2}\left(a \psi_{0}+h_{1}\right)+a_{2}\left|b \phi_{0}+h_{2}\right|^{2}\left(a \psi_{0}+h_{1}\right)\right\rangle,\right. \\
&\left.\left\langle\phi_{0}, a_{1}\left|b \phi_{0}+h_{2}\right|^{2}\left(b \phi_{0}+h_{2}\right)+a_{2}\left|a \psi_{0}+h_{1}\right|^{2}\left(b \phi_{0}+h_{2}\right)\right\rangle\right)^{T} .
\end{aligned}
$$

Now we have the following result on the center manifold of the system (1.1)-(1.2):

Theorem 2.1 Let $\delta_{E_{1}}, \delta_{E_{2}}, \delta>0$. Then for $\left|E_{1}-\omega_{1}\right|<\delta_{E_{1}},\left|E_{2}-\omega_{2}\right|<\delta_{E_{2}},\left\|\psi_{E_{1}}\right\|_{\mathbf{L}_{\sigma}^{2} \cap \mathbf{H}^{2}}<\delta$, and $\left\|\phi_{E_{2}}\right\|_{\mathbf{L}_{\sigma}^{2} \cap \mathbf{H}^{2}}<\delta$, there exist $\mathbf{C}^{1}$ functions

$$
\begin{aligned}
& h_{1}:\{a \in \mathbb{C}:|a|<\delta\} \mapsto \mathbf{L}_{\sigma}^{2} \cap \mathbf{H}^{2}, \\
& h_{2}:\{b \in \mathbb{C}:|b|<\delta\} \mapsto \mathbf{L}_{\sigma}^{2} \cap \mathbf{H}^{2}
\end{aligned}
$$

such that the eigenvalue problem (1.3)-(1.4) has a unique solution up to multiplication with $e^{i \theta}, \theta \in(0,2 \pi)$, which can be represented as

$$
\begin{array}{lll}
\psi_{E_{1}}=a \psi_{0}+h_{1}(a), & \left\langle\psi_{0}, h_{1}\right\rangle=0, & |a|<\delta, \\
\phi_{E_{2}}=b \phi_{0}+h_{2}(b), & \left\langle\phi_{0}, h_{2}\right\rangle=0, & |b|<\delta .
\end{array}
$$

Proof We follow the idea of $[8,9]$ and use the implicit function theorem to solve (2.1)-(2.2). We define a $\mathbf{C}^{1}$ function $\mathcal{F}:(-\infty, 0)^{2} \times \mathbb{C}^{2} \times\left(\mathbf{L}_{\sigma}^{2} \cap \mathbf{H}^{2}\right)^{2}$ as

$$
\mathcal{F}(\mathcal{E}, a, b, X)=X+(\mathcal{A}-\mathcal{E})^{-1} P_{c} F_{1}
$$

It is easy to see that

$$
\mathcal{F}\left(\mathcal{E}_{0}, 0,0,0\right)=0, \quad D_{X} \mathcal{F}\left(\mathcal{E}_{0}, 0,0,0\right)=I
$$

Therefore, from the implicit function theorem, we know that (2.1) has a unique solution $X=\bar{X}(\mathcal{E}, a, b)$ with $\|X\|_{\left(\mathbf{L}_{\sigma}^{2} \cap \mathbf{H}^{2}\right)^{2}}<\delta_{1}$, where $\bar{X}(\mathcal{E}, a, b)$ is a $\mathbf{C}^{1}$ function from $\left(\omega_{1}-\delta_{1}, \omega_{1}+\right.$ $\left.\delta_{1}\right) \times\left(\omega_{2}-\delta_{1}, \omega_{2}+\delta_{1}\right) \times\left\{(a, b) \in \mathbb{C}^{2}:|a|,|b|<\delta_{1}\right\}$ to $\left(\mathbf{L}_{\sigma}^{2} \cap \mathbf{H}^{2}\right)^{2}$ for $\delta_{1}>0$. By direct computation, we know that $\left(e^{i \theta} a, e^{i \theta} b, e^{i \theta} X\right)$ is also a solution of (2.1) for $\theta \in(0,2 \pi)$. By uniqueness, we have $\bar{X}(\mathcal{E}, a, b)=\tilde{\mathcal{A}} \bar{X}(\mathcal{E},|a|,|b|)$, where $\tilde{\mathcal{A}}=\operatorname{diag}\left(\frac{a}{|a|}, \frac{b}{|b|}\right)$.

Substituting $X=\bar{X}(\mathcal{E}, a, b)$ into (2.2). From $\bar{X}(\mathcal{E}, a, b)=\tilde{\mathcal{A}} \bar{X}(\mathcal{E},|a|,|b|)$, we have

$$
\mathcal{E}_{0}-\mathcal{E}=-\overline{\mathcal{B}}^{-1} F_{2}(|a|,|b|, \bar{X}(\mathcal{E},|a|,|b|)),
$$

where

$$
\overline{\mathcal{B}}=\left(\begin{array}{cc}
|a| & 0 \\
0 & |b|
\end{array}\right) .
$$

Define a $\mathbf{C}^{1}$ function $\mathcal{G}$ on $\left(\omega_{1}-\delta_{1}, \omega_{1}+\delta_{1}\right) \times\left(\omega_{2}-\delta_{1}, \omega_{2}+\delta_{1}\right) \times\left(-\delta_{1}, \delta_{1}\right)^{2}$ as follows:

$$
\mathcal{G}(\mathcal{E}, a, b)=\mathcal{E}_{0}-\mathcal{E}+\overline{\mathcal{B}}^{-1} F_{2}(|a|,|b|, \bar{X}(\mathcal{E},|a|,|b|)) .
$$


We can see that

$$
\mathcal{G}\left(\mathcal{E}_{0}, 0,0\right)=0, \quad D_{\mathcal{E}} \mathcal{F}\left(\mathcal{E}_{0}, 0,0\right)=-I .
$$

From the implicit function theorem, we find that (2.2) has a unique solution $\mathcal{E}=\tilde{\mathcal{E}}(|a|,|b|)$ with $X=\bar{X}(\mathcal{E}, a, b)$, where $\tilde{\mathcal{E}}(|a|,|b|)$ is a $\mathbf{C}^{1}$ function from $(-\delta, \delta) \times(-\delta, \delta)$ to $\left(\omega_{1}-\delta_{E_{1}}, \omega_{1}+\right.$ $\left.\delta_{E_{1}}\right) \times\left(\omega_{2}-\delta_{E_{2}}, \omega_{2}+\delta_{E_{2}}\right)$ for $0<\delta \leq \delta_{1}$ and $0<\delta_{E_{1}}, \delta_{E_{2}} \leq \delta_{1}$.

\section{Proof of Theorem 1.1}

We consider the asymptotic stability of the coupled Schrödinger system (1.1)-(1.2) in this section. The global well-posedness for the system (1.1)-(1.2) with small initial data is obtained by Cazenave [10].

Define

$$
a(t)=\left\langle\psi_{0}, u\right\rangle, \quad b(t)=\left\langle\phi_{0}, v\right\rangle, \quad \text { for } t \in \mathbb{R} .
$$

By Theorem 2.1, we can define $h_{1}(a(t))$ and $h_{2}(b(t)) \in \mathbb{C}$ by choosing $\varepsilon_{0}<\delta$. Therefore,

$$
\begin{array}{ll}
r_{1}(t)=u(t)-h_{1}(a(t))-a(t) \psi_{0}, & \left\langle\psi_{0}, r_{1}(t)\right\rangle \equiv 0, \\
r_{2}(t)=v(t)-h_{2}(b(t))-b(t) \phi_{0}, & \left\langle\phi_{0}, r_{2}(t)\right\rangle \equiv 0 .
\end{array}
$$

We can see that the solution is described by the scalar $a(t), b(t) \in \mathbb{C}$ and $r_{1}(t), r_{2}(t) \in$ $\mathbf{C}\left(\mathbb{R}, \mathbf{H}^{1}\right)$. More precisely, from (1.6)-(1.7), we have

$$
\begin{aligned}
i \frac{\mathrm{d} a}{\mathrm{~d} t} \psi_{0} & +i D h_{1} \mid \frac{\mathrm{d} a}{a}+i \frac{\mathrm{d} r_{1}}{\mathrm{~d} t} \\
= & E_{1} a \psi_{0}+E_{1} h_{1}(a)+\left(-\Delta+V_{1}\right) r_{1}+a_{1}\left(2\left|\psi_{E_{1}}\right|^{2} r_{1}+\psi_{E_{1}}^{2} \bar{r}_{1}\right. \\
& \left.+2 \psi_{E_{1}}\left|r_{1}\right|^{2}+\psi_{E_{1}} r_{1}^{2}+\left|r_{1}\right|^{2} r_{1}\right)+a_{2}\left(\phi_{E_{2}} \psi_{E_{1}} \bar{r}_{2}+\left|\phi_{E_{2}}\right|^{2} r_{1}\right. \\
& \left.+\phi_{E_{2}} r_{1} \bar{r}_{2}+\psi_{E_{1}} \bar{\phi}_{E_{2}} r_{2}+\left|r_{2}\right|^{2} \psi_{E_{1}}+\bar{\phi}_{E_{2}} r_{1} r_{2}+\left|r_{2}\right|^{2} r_{1}\right), \\
i \frac{\mathrm{d} b}{\mathrm{~d} t} \phi_{0} & +i D h_{2} \mid \frac{\mathrm{d} b}{\mathrm{~d} t}+i \frac{\mathrm{d} r_{2}}{\mathrm{~d} t} \\
= & E_{2} b \phi_{0}+E_{2} h_{2}(b)+\left(-\Delta+V_{3}\right) r_{2}+a_{3}\left(2\left|\phi_{E_{2}}\right|^{2} r_{2}+\phi_{E_{2}}^{2} \bar{r}_{2}\right. \\
& \left.+2 \phi_{E_{2}}\left|r_{2}\right|^{2}+\phi_{E_{2}} r_{2}^{2}+\left|r_{2}\right|^{2} r_{2}\right)+a_{4}\left(\phi_{E_{2}} \psi_{E_{1}} \bar{r}_{1}+\left|\psi_{E_{1}}\right|^{2} r_{2}\right. \\
& \left.+\psi_{E_{1}} r_{2} \bar{r}_{1}+\phi_{E_{2}} \bar{\psi}_{E_{1}} r_{1}+\left|r_{1}\right|^{2} \phi_{E_{2}}+\bar{\psi}_{E_{1}} r_{1} r_{2}+\left|r_{1}\right|^{2} r_{2}\right) .
\end{aligned}
$$

Note that $h_{1}, h_{2}$, and $D h_{1}, D h_{2}$ have range orthogonal to $\psi_{0}, \phi_{0} . r_{1}, r_{2}$ and $\frac{d r_{1}}{d t}$, $\frac{d r_{2}}{d t}$ are orthogonal to $\psi_{0}, \phi_{0}$, respectively. Therefore, from (3.1)-(3.2), we get

$$
\begin{aligned}
i \frac{\mathrm{d} a}{\mathrm{~d} t}= & E_{1}(|a|) a+a_{1}\left\langle\psi_{0}, 2\left|\psi_{E_{1}}\right|^{2} r_{1}+\psi_{E_{1}}^{2} \bar{r}_{1}+2 \psi_{E_{1}}\left|r_{1}\right|^{2}\right. \\
& \left.+\psi_{E_{1}} r_{1}^{2}+\left|r_{1}\right|^{2} r_{1}\right\rangle+a_{2}\left\langle\psi_{0}, \phi_{E_{2}} \psi_{E_{1}} \bar{r}_{2}+\left|\phi_{E_{2}}\right|^{2} r_{1}\right. \\
& \left.+\phi_{E_{2}} r_{1} \bar{r}_{2}+\psi_{E_{1}} \bar{\phi}_{E_{2}} r_{2}+\left|r_{2}\right|^{2} \psi_{E_{1}}+\bar{\phi}_{E_{2}} r_{1} r_{2}+\left|r_{2}\right|^{2} r_{1}\right\rangle,
\end{aligned}
$$




$$
\begin{aligned}
i \frac{\mathrm{d} b}{\mathrm{~d} t}= & E_{2}(|b|) b+\left.a_{3}\left|\phi_{0}, 2\right| \phi_{E_{2}}\right|^{2} r_{2}+\phi_{E_{2}}^{2} \bar{r}_{2}+2 \phi_{E_{2}}\left|r_{2}\right|^{2} \\
& \left.+\phi_{E_{2}} r_{2}^{2}+\left|r_{2}\right|^{2} r_{2}\right\rangle+\left.a_{4}\left|\phi_{0}, \phi_{E_{2}} \psi_{E_{1}} \bar{r}_{1}+\right| \psi_{E_{1}}\right|^{2} r_{2} \\
& \left.+\psi_{E_{1}} r_{2} \bar{r}_{1}+\phi_{E_{2}} \bar{\psi}_{E_{1}} r_{1}+\left|r_{1}\right|^{2} \phi_{E_{2}}+\bar{\psi}_{E_{1}} r_{1} r_{2}+\left|r_{1}\right|^{2} r_{2}\right\rangle .
\end{aligned}
$$

Using $\left.D h_{1}\right|_{a}\left(i E_{1} a\right)=-E_{1} i h_{1}(a)$ and $\left.D h_{2}\right|_{b}\left(i E_{2} b\right)=-E_{2} i h_{2}(b)$, we have

$$
\begin{aligned}
& i \frac{\mathrm{d} r_{1}}{\mathrm{~d} t}=\left(-\triangle+V_{1}\right) r_{1}+a_{1} P_{c}\left(2\left|\psi_{E_{1}}\right|^{2} r_{1}+\psi_{E_{1}}^{2} \bar{r}_{1}\right. \\
& \left.+2 \psi_{E_{1}}\left|r_{1}\right|^{2}+\psi_{E_{1}} r_{1}^{2}+\left|r_{1}\right|^{2} r_{1}\right)+a_{2} P_{c}\left(\phi_{E_{2}} \psi_{E_{1}} \bar{r}_{2}+\left|\phi_{E_{2}}\right|^{2} r_{1}\right. \\
& \left.+\phi_{E_{2}} r_{1} \bar{r}_{2}+\psi_{E_{1}} \bar{\phi}_{E_{2}} r_{2}+\left|r_{2}\right|^{2} \psi_{E_{1}}+\bar{\phi}_{E_{2}} r_{1} r_{2}+\left|r_{2}\right|^{2} r_{1}\right) \text {, } \\
& -\left.D h_{1}\right|_{a}\left(\left.a_{1}\left|\psi_{0}, 2\right| \psi_{E_{1}}\right|^{2} r_{1}+\psi_{E_{1}}^{2} \bar{r}_{1}+2 \psi_{E_{1}}\left|r_{1}\right|^{2}\right. \\
& \left.+\psi_{E_{1}} r_{1}^{2}+\left|r_{1}\right|^{2} r_{1}\right\rangle+a_{2}\left\langle\psi_{0}, \phi_{E_{2}} \psi_{E_{1}} \bar{r}_{2}+\left|\phi_{E_{2}}\right|^{2} r_{1}\right. \\
& \left.+\phi_{E_{2}} r_{1} \bar{r}_{2}+\psi_{E_{1}} \bar{\phi}_{E_{2}} r_{2}+\left|r_{2}\right|^{2} \psi_{E_{1}}+\bar{\phi}_{E_{2}} r_{1} r_{2}+\left|r_{2}\right|^{2} r_{1} \mid\right) \text {, } \\
& i \frac{\mathrm{d} r_{2}}{\mathrm{~d} t}=\left(-\triangle+V_{3}\right) r_{2}+a_{3} P_{c}\left(2\left|\phi_{E_{2}}\right|^{2} r_{2}+\phi_{E_{2}}^{2} \bar{r}_{2}\right. \\
& \left.+2 \phi_{E_{2}}\left|r_{2}\right|^{2}+\phi_{E_{2}} r_{2}^{2}+\left|r_{2}\right|^{2} r_{2}\right)+a_{4} P_{c}\left(\phi_{E_{2}} \psi_{E_{1}} \bar{r}_{1}+\left|\psi_{E_{1}}\right|^{2} r_{2}\right. \\
& \left.+\psi_{E_{1}} r_{2} \bar{r}_{1}+\phi_{E_{2}} \bar{\psi}_{E_{1}} r_{1}+\left|r_{1}\right|^{2} \phi_{E_{2}}+\bar{\psi}_{E_{1}} r_{1} r_{2}+\left|r_{1}\right|^{2} r_{2}\right) \\
& -\left.D h_{2}\right|_{b}\left(\left.a_{3}\left|\phi_{0}, 2\right| \phi_{E_{2}}\right|^{2} r_{2}+\phi_{E_{2}}^{2} \bar{r}_{2}+2 \phi_{E_{2}}\left|r_{2}\right|^{2}\right. \\
& \left.+\phi_{E_{2}} r_{2}^{2}+\left|r_{2}\right|^{2} r_{2}\right\rangle+\left.a_{4}\left|\phi_{0}, \phi_{E_{2}} \psi_{E_{1}} \bar{r}_{1}+\right| \psi_{E_{1}}\right|^{2} r_{2} \\
& \left.+\psi_{E_{1}} r_{2} \bar{r}_{1}+\phi_{E_{2}} \bar{\psi}_{E_{1}} r_{1}+\left|r_{1}\right|^{2} \phi_{E_{2}}+\bar{\psi}_{E_{1}} r_{1} r_{2}+\left|r_{1}\right|^{2} r_{2} \mid\right) \text {. }
\end{aligned}
$$

The linear part of (3.5)-(3.6) is

$$
\begin{aligned}
i \frac{\mathrm{d} r_{1}^{\prime}}{\mathrm{d} t}= & \left(-\Delta+V_{1}\right) r_{1}^{\prime}+a_{1} P_{c}\left(2\left|\psi_{E_{1}}\right|^{2} r_{1}^{\prime}+\psi_{E_{1}}^{2} \bar{r}_{1}^{\prime}\right) \\
& +a_{2} P_{c}\left(\phi_{E_{2}} \psi_{E_{1}} \bar{r}_{2}^{\prime}+\left|\phi_{E_{2}}\right|^{2} r_{1}^{\prime}+\psi_{E_{1}} \bar{\phi}_{E_{2}} r_{2}^{\prime}\right) \\
& -D h_{1}|a| \psi_{0}, a_{1}\left(2\left|\psi_{E_{1}}\right|^{2} r_{1}^{\prime}+\psi_{E_{1}}^{2} \bar{r}_{1}^{\prime}\right) \\
& \left.+a_{2}\left(\phi_{E_{2}} \psi_{E_{1}} \bar{r}_{2}^{\prime}+\left|\phi_{E_{2}}\right|^{2} r_{1}^{\prime}+\psi_{E_{1}} \bar{\phi}_{E_{2}} r_{2}^{\prime}\right)\right\rangle, \\
i \frac{\mathrm{d} r_{2}^{\prime}}{\mathrm{d} t=} & \left(-\Delta+V_{3}\right) r_{2}^{\prime}+a_{3} P_{c}\left(2\left|\phi_{E_{2}}\right|^{2} r_{2}^{\prime}+\phi_{E_{2}}^{2} \bar{r}_{2}^{\prime}\right) \\
& +a_{4} P_{c}\left(\phi_{E_{2}} \psi_{E_{1}} \bar{r}_{1}^{\prime}+\left|\psi_{E_{1}}\right|^{2} r_{2}^{\prime}+\phi_{E_{2}} \bar{\psi}_{E_{1}} r_{1}^{\prime}\right) \\
& -D h_{2}\left|{ }_{a}\right| \phi_{0}, a_{3}\left(2\left|\phi_{E_{2}}\right|^{2} r_{2}^{\prime}+\phi_{E_{2}}^{2} \bar{r}_{2}^{\prime}\right) \\
& \left.+a_{4}\left(\phi_{E_{2}} \psi_{E_{1}} \bar{r}_{1}^{\prime}+\left|\psi_{E_{1}}\right|^{2} r_{2}+\phi_{E_{2}} \bar{\psi}_{E_{1}} r_{1}^{\prime}\right)\right\rangle .
\end{aligned}
$$

Define the operator $\mathcal{S}(t, s) Y$ as the solution of the linear equation (3.7)-(3.8):

$$
\mathcal{S}(t, s) Y=R^{\prime} \quad \text { with } R^{\prime}=\left(r_{1}^{\prime}, r_{2}^{\prime}\right)^{T} .
$$

The following lemma can be obtained by a small modification of the proof of Theorem 4.1 in [8], so we omit the proof. 
Lemma 3.1 There exists $\varepsilon>0$ such that if

$$
\left\|\langle x\rangle^{\sigma} \psi_{E_{1}}\right\|_{\mathbf{H}^{2}}<\varepsilon, \quad\left\|\langle x\rangle^{\sigma} \phi_{E_{2}}\right\|_{\mathbf{H}^{2}}<\varepsilon,
$$

then there exist $C, C_{p}>0$ with the property that for any $t, s \in \mathbb{R}$, we have

$$
\begin{aligned}
& \|\mathcal{S}(t, s)\|_{\mathbf{L}_{\sigma}^{2} \times \mathbf{L}_{\sigma}^{2} \rightarrow \mathbf{L}_{-\sigma}^{2} \times \mathbf{L}_{-\sigma}^{2}} \leq C(1+|t-s|)^{-1} \log ^{-2}(2+|t-s|), \\
& \|\mathcal{S}(t, s)\|_{\mathbf{L}^{p^{\prime}} \times \mathbf{L}^{p^{\prime}} \rightarrow \mathbf{L}_{-\sigma}^{2} \times \mathbf{L}_{-\sigma}^{2}} \leq C_{p}|t-s|^{1-\frac{2}{p}} \\
& \|\mathcal{S}(t, s)\|_{\mathbf{L}_{\sigma}^{2} \times \mathbf{L}_{\sigma}^{2} \rightarrow \mathbf{L}^{p} \times \mathbf{L}^{p}} \leq C_{p}|t-s|^{1-\frac{2}{p}}
\end{aligned}
$$

where $p \geq 2$ and $\frac{1}{p^{\prime}}+\frac{1}{p}=1$.

By Duhamel?s principle, it follows from B.5)-(3.6) that

$$
R(t)=\mathcal{S}(t, 0) R(0)-i \int_{0}^{t} \mathcal{S}(t, s) P_{c} G_{1} d s+i \int_{0}^{t} \mathcal{S}(t, s)(D H) G_{2} d s,
$$

where $R=\left(r_{1}, r_{2}\right)^{T}, G_{1}=\left(g_{1}, g_{2}\right)^{T}, G_{2}=\left(g_{3}, g_{4}\right)^{T}$, and $D H=\operatorname{diag}\left(\left.D h_{1}\right|_{a},\left.D h_{2}\right|_{b}\right)$ with

$$
\begin{aligned}
g_{1}= & a_{1}\left(2 \psi_{E_{1}}\left|r_{1}\right|^{2}+\psi_{E_{1}} r_{1}^{2}+\left|r_{1}\right|^{2} r_{1}\right) \\
& +a_{2}\left(\phi_{E_{2}} r_{1} \bar{r}_{2}+\left|r_{2}\right|^{2} \psi_{E_{1}}+\bar{\phi}_{E_{2}} r_{1} r_{2}+\left|r_{2}\right|^{2} r_{1}\right), \\
g_{2}= & a_{3}\left(2 \phi_{E_{2}}\left|r_{2}\right|^{2}+\phi_{E_{2}} r_{2}^{2}+\left|r_{2}\right|^{2} r_{2}\right) \\
& +a_{4}\left(\psi_{E_{1}} r_{2} \bar{r}_{1}+\left|r_{1}\right|^{2} \phi_{E_{2}}+\bar{\psi}_{E_{1}} r_{1} r_{2}+\left|r_{1}\right|^{2} r_{2}\right), \\
g_{3}= & a_{1}\left\langle\psi_{0}, 2 \psi_{E_{1}}\left|r_{1}\right|^{2}+\psi_{E_{1}} r_{1}^{2}+\left|r_{1}\right|^{2} r_{1}\right\rangle \\
& +a_{2}\left\langle\psi_{0}, \phi_{E_{2}} r_{1} \bar{r}_{2}+\left|r_{2}\right|^{2} \psi_{E_{1}}+\bar{\phi}_{E_{2}} r_{1} r_{2}+\left|r_{2}\right|^{2} r_{1}\right\rangle, \\
g_{4}= & a_{3}\left\langle\phi_{0}, 2 \phi_{E_{2}}\left|r_{2}\right|^{2}+\phi_{E_{2}} r_{2}^{2}+\left|r_{2}\right|^{2} r_{2}\right\rangle \\
& +a_{4}\left\langle\phi_{0}, \psi_{E_{1}} r_{2} \bar{r}_{1}+\left|r_{1}\right|^{2} \phi_{E_{2}}+\bar{\psi}_{E_{1}} r_{1} r_{2}+\left|r_{1}\right|^{2} r_{2}\right\rangle .
\end{aligned}
$$

For fixed $p \geq 6$, we define the Banach space

$$
\begin{aligned}
\mathbb{B}= & \left\{u: \mathbb{R} \rightarrow \mathbf{L}_{-\sigma}^{2} \cap \mathbf{L}^{p} \cap \mathbf{L}^{2} \mid \sup _{t \geq 0}(1+t)^{1-\frac{2}{p}}\|u\|_{\mathbf{L}_{-\sigma}^{2}},\right. \\
& \sup _{t \geq 0} \frac{(1+|t|)^{1-\frac{2}{p}}}{\log (2+|t|)}\|u\|_{\left.\mathbf{L}^{p}, \sup _{t \geq 0}\|u\|_{\mathbf{L}^{2}}<\infty\right\}}
\end{aligned}
$$

endowed with the norm

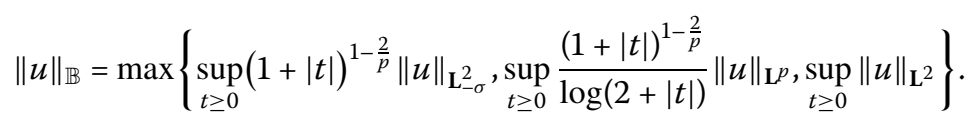

Consider the nonlinear part in (3.12):

$$
(\mathcal{N} R)(t):=-i \int_{0}^{t} \mathcal{S}(t, s) P_{c} G_{1} d s+i \int_{0}^{t} \mathcal{S}(t, s)(D H) G_{2} d s .
$$




\section{Lemma 3.2}

(1) For $R \in \mathbb{B} \times \mathbb{B}$, the nonlinear operator $\mathcal{N}: \mathbb{B} \times \mathbb{B} \rightarrow \mathbb{B} \times \mathbb{B}$ is well defined.

(2) We have

$$
\begin{aligned}
\left\|\mathcal{N} R_{1}-\mathcal{N} R_{2}\right\|_{\mathbb{B} \times \mathbb{B}} \leq & C_{a_{1}, a_{2}, a_{3}, a_{4}, p}\left(\left\|R_{1}\right\|_{\mathbb{B} \times \mathbb{B}}+\left\|R_{2}\right\|_{\mathbb{B} \times \mathbb{B}}+\left\|R_{1}\right\|_{\mathbb{B} \times \mathbb{B}}^{2}+\left\|R_{2}\right\|_{\mathbb{B} \times \mathbb{B}}^{2}\right) \\
& \times\left(\left\|R_{1}-R_{2}\right\|_{\mathbb{B} \times \mathbb{B}}\right) .
\end{aligned}
$$

Proof It is obvious that the part (1) can be obtained by part (2) choosing $R_{2}=0$. Now, we only need to prove (2). This proof is based on these estimates of $\mathcal{N} R_{1}-\mathcal{N} R_{2}$ : the $\mathbf{L}_{-\sigma}^{2} \times \mathbf{L}_{-\sigma}^{2}$ estimate, the $\mathbf{L}^{p} \times \mathbf{L}^{p}$ estimate and the $\mathbf{L}^{2} \times \mathbf{L}^{2}$ estimate. In fact, with a similar argument of getting the $\mathbf{L}_{-\sigma}^{2} \times \mathbf{L}_{-\sigma}^{2}$ estimate, we could obtain the $\mathbf{L}^{p} \times \mathbf{L}^{p}$ estimate and the $\mathbf{L}^{2} \times \mathbf{L}^{2}$ estimate from (3.10) and (3.11). Here, we consider the $\mathbf{L}_{-\sigma}^{2} \times \mathbf{L}_{-\sigma}^{2}$ estimate.

Let $R_{1}=\left(r_{1}, r_{2}\right)^{T}, R_{2}=\left(\tilde{r}_{1}, \tilde{r}_{2}\right)^{T} \in \mathbb{B} \times \mathbb{B}$. One obtains

$$
\begin{aligned}
& \left(\mathcal{N} R_{1}-\mathcal{N} R_{2}\right)(t) \\
& \quad=-i \int_{0}^{t} \mathcal{S}(t, s) P_{c}\left(G_{1}-\tilde{G}_{1}\right) d s+i \int_{0}^{t} \mathcal{S}(t, s)(D H)\left(G_{2}-\tilde{G}_{2}\right) d s,
\end{aligned}
$$

where $G_{1}-\tilde{G}_{1}=\left(g_{1}-\tilde{g}_{1}, g_{2}-\tilde{g}_{2}\right)^{T}$ and $G_{2}-\tilde{G}_{2}=\left(g_{3}-\tilde{g}_{3}, g_{4}-\tilde{g}_{4}\right)^{T}$ with

$$
\begin{aligned}
& g_{1}-\tilde{g}_{1}=2 a_{1} \psi_{E_{1}}\left(\left|r_{1}\right|-\left|\tilde{r}_{1}\right|\right)\left(\left|r_{1}\right|+\left|\tilde{r}_{1}\right|\right)+a_{1} \psi_{E_{1}}\left(r_{1}-\tilde{r}_{1}\right)\left(r_{1}+\tilde{r}_{1}\right) \\
& +a_{1}\left(\left|r_{1}\right|^{2}\left(r_{1}-\tilde{r}_{1}\right)+\left(\left|r_{1}\right|-\left|\tilde{r}_{1}\right|\right)\left(\tilde{r}_{1}\left|r_{1}\right|+\tilde{r}_{1}\left|\tilde{r}_{1}\right|\right)\right) \\
& +a_{2} \phi_{E_{2}}\left(\left(r_{1}-\tilde{r}_{1}\right) \bar{r}_{2}+\tilde{r}_{1}\left(\bar{r}_{2}-\tilde{\bar{r}}_{2}\right)\right)+a_{2} \psi_{E_{1}}\left(\left|r_{2}\right|-\left|\tilde{r}_{2}\right|\right)\left(\left|r_{2}\right|+\left|\tilde{r}_{2}\right|\right) \\
& +a_{2} \bar{\phi}_{E_{2}}\left(\left(r_{1}-\tilde{r}_{1}\right) r_{2}+\tilde{r}_{1}\left(r_{2}-\tilde{r}_{2}\right)\right) \\
& +a_{2}\left(\left(\left|r_{2}\right|-\left|\tilde{r}_{2}\right|\right)\left(\left|r_{2}\right|+\left|\tilde{r}_{2}\right|\right) r_{1}+\left|\tilde{r}_{2}\right|^{2}\left(r_{1}-\tilde{r}_{1}\right)\right), \\
& g_{2}-\tilde{g}_{2}=2 a_{3} \phi_{E_{2}}\left(\left|r_{2}\right|-\left|\tilde{r}_{2}\right|\right)\left(\left|r_{2}\right|+\left|\tilde{r}_{2}\right|\right)+a_{3} \phi_{E_{2}}\left(r_{2}-\tilde{r}_{2}\right)\left(r_{2}+\tilde{r}_{2}\right) \\
& +a_{3}\left(\left|r_{2}\right|^{2}\left(r_{2}-\tilde{r}_{2}\right)+\left(\left|r_{2}\right|-\left|\tilde{r}_{2}\right|\right)\left(\tilde{r}_{2}\left|r_{2}\right|+\tilde{r}_{2}\left|\tilde{r}_{2}\right|\right)\right) \\
& +a_{4} \psi_{E_{1}}\left(\left(r_{2}-\tilde{r}_{2}\right) \bar{r}_{1}+\tilde{r}_{2}\left(\bar{r}_{1}-\tilde{\bar{r}}_{1}\right)\right)+a_{4} \phi_{E_{2}}\left(\left|r_{1}\right|-\left|\tilde{r}_{1}\right|\right)\left(\left|r_{1}\right|+\left|\tilde{r}_{1}\right|\right) \\
& +a_{4} \bar{\psi}_{E_{1}}\left(\left(r_{2}-\tilde{r}_{2}\right) r_{1}+\tilde{r}_{2}\left(r_{1}-\tilde{r}_{1}\right)\right) \\
& +a_{4}\left(\left(\left|r_{1}\right|-\left|\tilde{r}_{1}\right|\right)\left(\left|r_{1}\right|+\left|\tilde{r}_{1}\right|\right) r_{2}+\left|\tilde{r}_{1}\right|^{2}\left(r_{2}-\tilde{r}_{2}\right)\right), \\
& g_{3}-\tilde{g}_{3}=a_{1}\left\langle\psi_{0}, 2 \psi_{E_{1}}\left(\left|r_{1}\right|-\left|\tilde{r}_{1}\right|\right)\left(\left|r_{1}\right|+\left|\tilde{r}_{1}\right|\right)+\psi_{E_{1}}\left(r_{1}-\tilde{r}_{1}\right)\left(r_{1}+\tilde{r}_{1}\right)\right\rangle \\
& +a_{1}\left\langle\psi_{0},\left|r_{1}\right|^{2}\left(r_{1}-\tilde{r}_{1}\right)+\left(\left|r_{1}\right|-\left|\tilde{r}_{1}\right|\right)\left(\tilde{r}_{1}\left|r_{1}\right|+\tilde{r}_{1}\left|\tilde{r}_{1}\right|\right)\right\rangle \\
& +a_{2}\left\langle\psi_{0}, \phi_{E_{2}}\left(\left(r_{1}-\tilde{r}_{1}\right) \bar{r}_{2}+\tilde{r}_{1}\left(\bar{r}_{2}-\tilde{\bar{r}}_{2}\right)\right)+\psi_{E_{1}}\left(\left|r_{2}\right|-\left|\tilde{r}_{2}\right|\right)\left(\left|r_{2}\right|+\left|\tilde{r}_{2}\right|\right)\right\rangle \\
& +a_{2}\left\langle\psi_{0}, \bar{\phi}_{E_{2}}\left(\left(r_{1}-\tilde{r}_{1}\right) r_{2}+\tilde{r}_{1}\left(r_{2}-\tilde{r}_{2}\right)\right)\right\rangle \\
& +a_{2}\left\langle\psi_{0},\left(\left|r_{2}\right|-\left|\tilde{r}_{2}\right|\right)\left(\left|r_{2}\right|+\left|\tilde{r}_{2}\right|\right) r_{1}+\left|\tilde{r}_{2}\right|^{2}\left(r_{1}-\tilde{r}_{1}\right)\right\rangle, \\
& g_{4}-\tilde{g}_{4}=a_{3}\left\langle\phi_{0}, 2 \phi_{E_{2}}\left(\left|r_{2}\right|-\left|\tilde{r}_{2}\right|\right)\left(\left|r_{2}\right|+\left|\tilde{r}_{2}\right|\right)+\phi_{E_{2}}\left(r_{2}-\tilde{r}_{2}\right)\left(r_{2}+\tilde{r}_{2}\right)\right\rangle \\
& +a_{3}\left\langle\phi_{0},\left|r_{2}\right|^{2}\left(r_{2}-\tilde{r}_{2}\right)+\left(\left|r_{2}\right|-\left|\tilde{r}_{2}\right|\right)\left(\tilde{r}_{2}\left|r_{2}\right|+\tilde{r}_{2}\left|\tilde{r}_{2}\right|\right)\right\rangle \\
& +a_{4}\left\langle\phi_{0}, \psi_{E_{1}}\left(\left(r_{2}-\tilde{r}_{2}\right) \bar{r}_{1}+\tilde{r}_{2}\left(\bar{r}_{1}-\tilde{\bar{r}}_{1}\right)\right)+\phi_{E_{2}}\left(\left|r_{1}\right|-\left|\tilde{r}_{1}\right|\right)\left(\left|r_{1}\right|+\left|\tilde{r}_{1}\right|\right)\right\rangle
\end{aligned}
$$




$$
\begin{aligned}
& +a_{4}\left\langle\phi_{0}, \bar{\psi}_{E_{1}}\left(\left(r_{2}-\tilde{r}_{2}\right) r_{1}+\tilde{r}_{2}\left(r_{1}-\tilde{r}_{1}\right)\right)\right\rangle \\
& +a_{4}\left\langle\phi_{0},\left(\left|r_{1}\right|-\left|\tilde{r}_{1}\right|\right)\left(\left|r_{1}\right|+\left|\tilde{r}_{1}\right|\right) r_{2}+\left|\tilde{r}_{1}\right|^{2}\left(r_{2}-\tilde{r}_{2}\right)\right\rangle .
\end{aligned}
$$

Let $4<p<\infty$ and $\frac{1}{p^{\prime}}+\frac{1}{p}=1$. We have

$$
\begin{aligned}
&\left\|\mathcal{N} R_{1}-\mathcal{N} R_{2}\right\|_{\mathbf{L}_{-\sigma}^{2} \times \mathbf{L}_{-\sigma}^{2}} \\
& \leq \int_{0}^{t}\|\mathcal{S}(t, s)\|_{\mathbf{L}_{\sigma}^{2} \times \mathbf{L}_{\sigma}^{2} \rightarrow \mathbf{L}_{-\sigma}^{2} \times \mathbf{L}_{-\sigma}^{2}}\|A\|_{\mathbf{L}^{2} \times \mathbf{L}^{2}} d s \\
& \quad+\int_{0}^{t}\|\mathcal{S}(t, s)\|_{\mathbf{L}_{\sigma}^{p^{\prime}} \times \mathbf{L}_{\sigma}^{p^{\prime}} \rightarrow \mathbf{L}_{-\sigma}^{2} \times \mathbf{L}_{-\sigma}^{2}}\|B\|_{\mathbf{L}^{p^{\prime}} \times \mathbf{L}^{p^{\prime}}} d s \\
& \quad+\int_{0}^{t}\|\mathcal{S}(t, s)\|_{\mathbf{L}_{\sigma}^{2} \times \mathbf{L}_{\sigma}^{2} \rightarrow \mathbf{L}_{-\sigma}^{2} \times \mathbf{L}_{-\sigma}^{2}}\|D H\|_{\mathbf{L}_{\sigma}^{2} \times \mathbf{L}_{\sigma}^{2}}\left|G_{2}-\tilde{G}_{2}\right| d s,
\end{aligned}
$$

where $A=\left(A_{1}, A_{2}\right)^{T}$ and $B=\left(B_{1}, B_{2}\right)^{T}$ with

$$
\begin{aligned}
A_{1}= & 2 a_{1} \psi_{E_{1}}\langle x\rangle^{\sigma}\left(\left|r_{1}\right|-\left|\tilde{r}_{1}\right|\right)\left(\left|r_{1}\right|+\left|\tilde{r}_{1}\right|\right)+a_{1} \psi_{E_{1}}\langle x\rangle^{\sigma}\left(r_{1}-\tilde{r}_{1}\right)\left(r_{1}+\tilde{r}_{1}\right) \\
& +a_{2} \phi_{E_{2}}\langle x\rangle^{\sigma}\left(\left(r_{1}-\tilde{r}_{1}\right) \bar{r}_{2}+\tilde{r}_{1}\left(\bar{r}_{2}-\tilde{\bar{r}}_{2}\right)\right)+a_{2} \psi_{E_{1}}\langle x\rangle^{\sigma}\left(\left|r_{2}\right|-\left|\tilde{r}_{2}\right|\right)\left(\left|r_{2}\right|+\left|\tilde{r}_{2}\right|\right) \\
& +a_{2} \bar{\phi}_{E_{2}}\langle x\rangle^{\sigma}\left(\left(r_{1}-\tilde{r}_{1}\right) r_{2}+\tilde{r}_{1}\left(r_{2}-\tilde{r}_{2}\right)\right), \\
A_{2}= & 2 a_{3} \phi_{E_{2}}\langle x\rangle^{\sigma}\left(\left|r_{2}\right|-\left|\tilde{r}_{2}\right|\right)\left(\left|r_{2}\right|+\left|\tilde{r}_{2}\right|\right)+a_{3} \phi_{E_{2}}\langle x\rangle^{\sigma}\left(r_{2}-\tilde{r}_{2}\right)\left(r_{2}+\tilde{r}_{2}\right) \\
& +a_{4} \psi_{E_{1}}\langle x\rangle^{\sigma}\left(\left(r_{2}-\tilde{r}_{2}\right) \bar{r}_{1}+\tilde{r}_{2}\left(\bar{r}_{1}-\tilde{\bar{r}}_{1}\right)\right)+a_{4} \phi_{E_{2}}\langle x\rangle^{\sigma}\left(\left|r_{1}\right|-\left|\tilde{r}_{1}\right|\right)\left(\left|r_{1}\right|+\left|\tilde{r}_{1}\right|\right) \\
& +a_{4} \bar{\psi}_{E_{1}}\langle x\rangle^{\sigma}\left(\left(r_{2}-\tilde{r}_{2}\right) r_{1}+\tilde{r}_{2}\left(r_{1}-\tilde{r}_{1}\right)\right), \\
B_{1}= & a_{1}\left(\left|r_{1}\right|^{2}\left(r_{1}-\tilde{r}_{1}\right)+\left(\left|r_{1}\right|-\left|\tilde{r}_{1}\right|\right)\left(\tilde{r}_{1}\left|r_{1}\right|+\tilde{r}_{1}\left|\tilde{r}_{1}\right|\right)\right) \\
& +a_{2}\left(\left(\left|r_{2}\right|-\left|\tilde{r}_{2}\right|\right)\left(\left|r_{2}\right|+\left|\tilde{r}_{2}\right|\right) r_{1}+\left|\tilde{r}_{2}\right|^{2}\left(r_{1}-\tilde{r}_{1}\right)\right), \\
B_{2}= & a_{3}\left(\left|r_{2}\right|^{2}\left(r_{2}-\tilde{r}_{2}\right)+\left(\left|r_{2}\right|-\left|\tilde{r}_{2}\right|\right)\left(\tilde{r}_{2}\left|r_{2}\right|+\tilde{r}_{2}\left|\tilde{r}_{2}\right|\right)\right) \\
& +a_{4}\left(\left(\left|r_{1}\right|-\left|\tilde{r}_{1}\right|\right)\left(\left|r_{1}\right|+\left|\tilde{r}_{1}\right|\right) r_{2}+\left|\tilde{r}_{1}\right|^{2}\left(r_{2}-\tilde{r}_{2}\right)\right) .
\end{aligned}
$$

In what follows, we consider the integral terms on $A, B$, and $\left|G_{2}-\tilde{G}_{2}\right|$ in (3.23). We first estimate the integral term on $A$. For $\frac{1}{\alpha}+\frac{2}{p}=\frac{1}{2}$, we have

$$
\left\|\psi_{E_{1}}\langle x\rangle^{\sigma}\left(\left|r_{1}\right|-\left|\tilde{r}_{1}\right|\right)\left(\left|r_{1}\right|+\left|\tilde{r}_{1}\right|\right)\right\|_{\mathbf{L}^{2}} \leq\left\|\psi_{E_{1}}\langle x\rangle^{\sigma}\right\|_{\mathbf{L}^{\alpha}}\left\|\left|r_{1}\right|-\left|\tilde{r}_{1}\right|\right\|_{\mathbf{L}^{2}}\left\|\left|r_{1}\right|+\left|\tilde{r}_{1}\right|\right\|_{\mathbf{L}^{2}} .
$$

From (3.9) in Lemma 3.1, we have

$$
\begin{aligned}
\int_{0}^{t} \| & \mathcal{S}(t, s)\left\|_{\mathbf{L}_{\sigma}^{2} \times \mathbf{L}_{\sigma}^{2} \rightarrow \mathbf{L}_{-\sigma}^{2} \times \mathbf{L}_{-\sigma}^{2}}\right\| A \|_{\mathbf{L}^{2} \times \mathbf{L}^{2}} d s \\
\leq & 3 C_{a_{1}, a_{2}, a_{3}, a_{4}} \int_{0}^{t}(1+|t-s|)^{-1} \log ^{-2}(2+|t-s|) \\
& \times\left\|\psi_{E_{1}}\langle x\rangle^{\sigma}\right\|_{\mathbf{L}^{\alpha}}\left\|\phi_{E_{2}}\langle x\rangle^{\sigma}\right\|_{\mathbf{L}^{\alpha}}\left(\left\|\left|r_{1}\right|-\left|\tilde{r}_{1}\right|\right\|_{\mathbf{L}^{2}}\left\|\left|r_{1}\right|+\left|\tilde{r}_{1}\right|\right\|_{\mathbf{L}^{2}}\right. \\
& \left.+\left\|\left|r_{2}\right|-\left|\tilde{r}_{2}\right|\right\|_{\mathbf{L}^{2}}\left\|\left|r_{2}\right|+\left|\tilde{r}_{2}\right|\right\|_{\mathbf{L}^{2}}\right) d s \\
\leq & 3 C_{a_{1}, a_{2}, a_{3}, a_{4}} C_{1} \int_{0}^{t} \frac{\log ^{2}(2+|s|)}{(1+|t-s|) \log (2+|t-s|)} \frac{\left\|\left|r_{1}\right|-\left|\tilde{r}_{1}\right|\right\|_{\mathbb{B}}+\left\|\left|r_{2}\right|-\left|\tilde{r}_{2}\right|\right\| \mathbb{B}}{(1+|s|)^{1-\frac{2}{p}}}
\end{aligned}
$$




$$
\begin{aligned}
& \times \frac{\left\|\left|r_{1}\right|+\left|\tilde{r}_{1}\right|\right\|_{\mathbb{B}}+\left\|\left|r_{2}\right|+\left|\tilde{r}_{2}\right|\right\|_{\mathbb{B}}}{(1+|s|)^{1-\frac{2}{p}}} d s \\
\leq & 3 C_{a_{1}, a_{2}, a_{3}, a_{4}} C_{1} C_{2}\left(\left\|r_{1}\right\|_{\mathbb{B}}+\left\|\tilde{r}_{1}\right\|_{\mathbb{B}}+\left\|r_{2}\right\|_{\mathbb{B}}+\left\|\tilde{r}_{2}\right\|_{\mathbb{B}}\right) \\
& \times \frac{\left\|r_{1}-\tilde{r}_{1}\right\|_{\mathbb{B}}+\left\|r_{2}-\tilde{r}_{2}\right\|_{\mathbb{B}}}{(1+|t|) \log ^{2}(2+|t|)},
\end{aligned}
$$

where the constants

$$
C_{1}=\max \left\{\sup _{t>0}\left\|\psi_{E_{1}}\langle x\rangle^{\sigma}\right\|_{\mathbf{L}^{\alpha}} \sup _{t>0}\left\|\phi_{E_{2}}\langle x\rangle^{\sigma}\right\|_{\mathbf{L}^{\alpha}}\right\}
$$

and

$$
C_{2}=\sup _{t>0}(1+|t|) \log ^{2}(2+|t|) \int_{0}^{t} \frac{\log ^{2}(2+|s|)}{(1+|t-s|) \log ^{2}(2+|t-s|)(1+|s|)^{2-\frac{4}{p}}} d s<\infty .
$$

Using the interpolate inequality $\|r\|_{\mathbf{L}^{\alpha}} \leq\|r\|_{\mathbf{L}^{2}}^{1-b}\|r\|_{\mathbf{L}^{p}}^{b}$ for $\frac{1}{\alpha}=\frac{1-b}{2}+\frac{b}{p}$ with $p \geq 4$ and $2 \leq \alpha \leq p$, one obtains

$$
\begin{aligned}
& \left\|\left|r_{1}\right|^{2}\left(r_{1}-\tilde{r}_{1}\right)\right\|_{\mathbf{L}^{p^{\prime}}} \leq\left\|r_{1}-\tilde{r}_{1}\right\|_{\mathbf{L}^{p}}\left\|r_{1}\right\|_{\mathbf{L}^{\alpha}}^{2} \\
& \leq\left\|r_{1}-\tilde{r}_{1}\right\|_{\mathbf{L}^{p}}\left\|r_{1}\right\|_{\mathbf{L}^{2}}^{2(1-b)}\left\|r_{1}\right\|_{\mathbf{L}^{p}}^{2 b}, \\
& \left\|\left(\left|r_{2}\right|-\left|\tilde{r}_{2}\right|\right)\left(\left|r_{2}\right|+\left|\tilde{r}_{2}\right|\right) r_{1}\right\|_{\mathbf{L}^{p^{\prime}}} \\
& \leq\left\|r_{1}-\tilde{r}_{1}\right\|_{\mathbf{L}^{p}}\left(\left\|r_{1}\right\|_{\mathbf{L}^{\alpha}}+\left\|\tilde{r}_{1}\right\|_{\mathbf{L}^{\alpha}}\right)\left\|r_{1}\right\|_{\mathbf{L}^{\alpha}} \\
& \leq\left\|r_{1}-\tilde{r}_{1}\right\|_{\mathbf{L}^{p}}\left(\left\|r_{1}\right\|_{\mathbf{L}^{2}}^{1-b}\left\|r_{1}\right\|_{\mathbf{L}^{p}}^{b}+\left\|\tilde{r}_{1}\right\|_{\mathbf{L}^{2}}^{1-b}\left\|\tilde{r}_{1}\right\|_{\mathbf{L}^{p}}^{b}\right)\left\|r_{1}\right\|_{\mathbf{L}^{2}}^{1-b}\left\|r_{1}\right\|_{\mathbf{L}^{p}}^{b}, \\
& \left\|\left(\left|r_{1}\right|-\left|\tilde{r}_{1}\right|\right)\left(\tilde{r}_{1}\left|r_{1}\right|+\tilde{r}_{1}\left|\tilde{r}_{1}\right|\right)\right\|_{\mathbf{L}^{p^{\prime}}} \\
& \leq\left\|r_{1}-\tilde{r}_{1}\right\|_{\mathbf{L}^{p}}\left\|\tilde{r}_{1}\right\|_{\mathbf{L}^{\alpha}}\left(\left\|r_{1}\right\|_{\mathbf{L}^{\alpha}}+\left\|\tilde{r}_{1}\right\|_{\mathbf{L}^{\alpha}}\right) \\
& \leq\left\|r_{1}-\tilde{r}_{1}\right\|_{\mathbf{L}^{p}}\left(\left\|r_{1}\right\|_{\mathbf{L}^{2}}^{1-b}\left\|r_{1}\right\|_{\mathbf{L}^{p}}^{b}+\left\|\tilde{r}_{1}\right\|_{\mathbf{L}^{2}}^{1-b}\left\|\tilde{r}_{1}\right\|_{\mathbf{L}^{p}}^{b}\right)\left\|\tilde{r}_{1}\right\|_{\mathbf{L}^{2}}^{1-b}\left\|\tilde{r}_{1}\right\|_{\mathbf{L}^{p}}^{b},
\end{aligned}
$$

where $\frac{2}{\alpha}+\frac{1}{p}=\frac{1}{p^{\prime}}$ and $\frac{2(1-b)}{2}+\frac{2 b}{p}+\frac{1}{p}=\frac{1}{p^{\prime}}$ with $\left(1-\frac{2}{p}\right)(1+2 b)=1+\frac{2}{p}>1$.

Note that $\left(1-\frac{2}{p}\right)(1+2 b)>1$. Therefore, from (3.10), we have

$$
\begin{aligned}
\int_{0}^{t}\|\mathcal{S}(t, s)\|_{\mathbf{L}_{\sigma}^{p^{\prime}} \times \mathbf{L}_{\sigma}^{p^{\prime}} \rightarrow \mathbf{L}_{-\sigma}^{2} \times \mathbf{L}_{-\sigma}^{2}}\|B\|_{\mathbf{L}^{p^{\prime}} \times \mathbf{L}^{p^{\prime}}} d s \\
\leq C_{p, a_{1}, a_{2}, a_{3}, a_{4}}\left(\left\|r_{1}\right\|_{\mathbb{B}}^{2}+\left\|\tilde{r}_{1}\right\|_{\mathbb{B}}^{2}+\left\|r_{2}\right\|_{\mathbb{B}}^{2}+\left\|\tilde{r}_{2}\right\|_{\mathbb{B}}^{2}\right) \\
\quad \times\left(\left\|r_{1}-\tilde{r}_{1}\right\|_{\mathbb{B}}+\left\|r_{2}-\tilde{r}_{2}\right\|_{\mathbb{B}}\right) \int_{0}^{t} \frac{\log (2+|s|)^{1+2 b}}{|t-s|^{1-\frac{2}{p}}(1+|s|)^{\left(1-\frac{2}{p}\right)(1+2 b)}} d s \\
\leq C_{p, a_{1}, a_{2}, a_{3}, a_{4}} C_{3}\left(\left\|r_{1}\right\|_{\mathbb{B}}^{2}+\left\|\tilde{r}_{1}\right\|_{\mathbb{B}}^{2}+\left\|r_{2}\right\|_{\mathbb{B}}^{2}+\left\|\tilde{r}_{2}\right\|_{\mathbb{B}}^{2}\right) \frac{\left\|r_{1}-\tilde{r}_{1}\right\|_{\mathbb{B}}+\left\|r_{2}-\tilde{r}_{2}\right\|_{\mathbb{B}}}{(1+|s|)^{1-\frac{2}{p}}},
\end{aligned}
$$

where the constant

$$
C_{3}=\sup _{t>0}(1+|t|)^{1-\frac{2}{p}} \int_{0}^{t} \frac{\log (2+|s|)^{1+2 b}}{|t-s|^{1-\frac{2}{p}}(1+|s|)^{\left(1-\frac{2}{p}\right)(1+2 b)}} d s<\infty .
$$


It is easy to obtain

$$
\begin{aligned}
& \left|\left\langle\psi_{0}, 2 \psi_{E_{1}}\left(\left|r_{1}\right|-\left|\tilde{r}_{1}\right|\right)\left(\left|r_{1}\right|+\left|\tilde{r}_{1}\right|\right)\right\rangle\right| \leq 2\left\|\psi_{0}\right\|_{\mathbf{L}^{\infty}}\left\|\psi_{E_{1}}\right\|_{\mathbf{L}^{\alpha}}\left\|r_{1}-\tilde{r}_{1}\right\|_{\mathbf{L}^{p}}\left(\left\|r_{1}\right\|_{\mathbf{L}^{p}}+\left\|\tilde{r}_{1}\right\|_{\mathbf{L}^{p}}\right), \\
& \left|\left\langle\psi_{0},\left|r_{1}\right|^{2}\left(r_{1}-\tilde{r}_{1}\right)\right\rangle\right| \leq\left\|\psi_{0}\langle x\rangle^{\sigma}\right\|_{\mathbf{L}^{\alpha}}\left\|r_{1}-\tilde{r}_{1}\right\|_{\mathbf{L}_{-\sigma}^{2}}\left\|r_{1}\right\|_{\mathbf{L}^{p}}^{2}
\end{aligned}
$$

for $\frac{1}{\alpha}+\frac{2}{p}=\frac{1}{2}$. From (3.9) in Lemma 3.1, we get

$$
\begin{aligned}
\int_{0}^{t}\|\mathcal{S}(t, s)\|_{\mathbf{L}_{\sigma}^{2} \times \mathbf{L}_{\sigma}^{2} \rightarrow \mathbf{L}_{-\sigma}^{2} \times \mathbf{L}_{-\sigma}^{2}}\left|G_{2}-\tilde{G}_{2}\right| d s \\
\leq C_{a_{1}, a_{2}, a_{3}, a_{4}}\left(\left\|r_{1}\right\|_{\mathbb{B}}+\left\|\tilde{r}_{1}\right\|_{\mathbb{B}}+\left\|r_{2}\right\|_{\mathbb{B}}+\left\|\tilde{r}_{2}\right\|_{\mathbb{B}}+\left\|r_{1}\right\|_{\mathbb{B}}^{2}+\left\|\tilde{r}_{1}\right\|_{\mathbb{B}}^{2}+\left\|r_{2}\right\|_{\mathbb{B}}^{2}+\left\|\tilde{r}_{2}\right\|_{\mathbb{B}}^{2}\right) \\
\quad \times\left(\left\|r_{1}-\tilde{r}_{1}\right\|_{\mathbb{B}}+\left\|r_{2}-\tilde{r}_{2}\right\|_{\mathbb{B}}\right) \int_{0}^{t} \frac{\left(\left\|\psi_{0}\langle x\rangle^{\sigma}\right\|_{\mathbf{L}^{\alpha}}+\left\|\phi_{0}\langle x\rangle^{\sigma}\right\|_{\mathbf{L}^{\alpha}}\right)}{(1+|t-s|) \log ^{2}(2+|t-s|)} \cdot \frac{\log ^{2}(2+|s|)}{(1+|s|)^{3-\frac{6}{p}}} d s \\
\leq C_{a_{1}, a_{2}, a_{3}, a_{4}} C_{5}\left(\left\|r_{1}\right\|_{\mathbb{B}}+\left\|\tilde{r}_{1}\right\|_{\mathbb{B}}+\left\|r_{2}\right\|_{\mathbb{B}}+\left\|\tilde{r}_{2}\right\|_{\mathbb{B}}+\left\|r_{1}\right\|_{\mathbb{B}}^{2}+\left\|\tilde{r}_{1}\right\|_{\mathbb{B}}^{2}+\left\|r_{2}\right\|_{\mathbb{B}}^{2}+\left\|\tilde{r}_{2}\right\|_{\mathbb{B}}^{2}\right) \\
\quad \times \frac{\left\|r_{1}-\tilde{r}_{1}\right\|_{\mathbb{B}}+\left\|r_{2}-\tilde{r}_{2}\right\|_{\mathbb{B}}}{(1+|t|) \log ^{2}(2+|t|)},
\end{aligned}
$$

where we have the constant

$$
C_{5}=\left(\left\|\psi_{0}\langle x\rangle^{\sigma}\right\|_{\mathbf{L}^{\alpha}}+\left\|\phi_{0}\langle x\rangle^{\sigma}\right\|_{\mathbf{L}^{\alpha}}\right) \int_{0}^{t} \frac{\log ^{2}(2+|s|)}{(1+|s|)^{3-\frac{6}{p}}(1+|t-s|) \log ^{2}(2+|t-s|)} d s<\infty .
$$

Hence, we conclude that

$$
\begin{aligned}
&\left\|\mathcal{N} R_{1}-\mathcal{N} R_{2}\right\|_{\mathbb{B} \times \mathbb{B}} \\
& \leq C_{a_{1}, a_{2}, a_{3}, a_{4}, p}\left(\left\|r_{1}\right\|_{\mathbb{B}}+\left\|\tilde{r}_{1}\right\|_{\mathbb{B}}+\left\|r_{2}\right\|_{\mathbb{B}}+\left\|\tilde{r}_{2}\right\|_{\mathbb{B}}+\left\|r_{1}\right\|_{\mathbb{B}}^{2}+\left\|\tilde{r}_{1}\right\|_{\mathbb{B}}^{2}\right. \\
&\left.+\left\|r_{2}\right\|_{\mathbb{B}}^{2}+\left\|\tilde{r}_{2}\right\|_{\mathbb{B}}^{2}\right) \times\left(\left\|r_{1}-\tilde{r}_{1}\right\|_{\mathbb{B}}+\left\|r_{2}-\tilde{r}_{2}\right\|_{\mathbb{B}}\right) .
\end{aligned}
$$

This completes the proof.

Proof of Theorem 1.1 Define a closed ball $B\left(R_{0}, \mathbf{r}\right) \subset \mathbb{B} \times \mathbb{B}$ with center $R_{0}=\mathcal{S}(t, 0) R(0)$ and radius $\mathbf{r}=\frac{L\left\|R_{0}\right\|_{\mathbb{B} \times \mathbb{B}}}{2-\text { Lip }}$. By Lemma 3.1, there exists a constant $C_{6}$ such that

$$
\left\|R_{0}\right\|_{\mathbb{B} \times \mathbb{B}} \leq C_{6}\|R(0)\|_{\mathbf{L}_{\sigma}^{2} \times \mathbf{L}_{\sigma}^{2}} .
$$

Choose $\varepsilon_{0}$ such that $C_{6} \varepsilon_{0}<\frac{1}{2}\left(\sqrt{1+2 C_{a_{1}, a_{2}, a_{3}, a_{4}, p}^{-1}}-1\right)$. Then there exists a constant $0<\mathrm{Lip}<$ 1 such that

$$
\left\|R_{0}\right\|_{\mathbb{B} \times \mathbb{B}} \leq \frac{2-\operatorname{Lip}}{4}\left(\sqrt{1+2 \operatorname{Lip} C_{a_{1}, a_{2}, a_{3}, a_{4}, p}^{-1}}-1\right) .
$$

It is easy to conclude that the right hand side of (3.12) leaves $B\left(R_{0}, \mathbf{r}\right)$ invariant, and it is a contraction with Lipschitz constant Lip on $B\left(R_{0}, \mathbf{r}\right)$. From the contraction mapping theorem, (3.12) has a unique solution in $B\left(R_{0}, \mathbf{r}\right)$. If we have two solutions of (3.12), one in $\mathbf{C}\left(\mathbb{R}, \mathbf{H}^{1} \times \mathbf{H}^{1}\right)$ from classical well-posedness theory and one in $\mathbf{C}\left(\mathbb{R},\left(\mathbf{L}_{-\sigma}^{2} \cap \mathbf{L}^{2} \cap \mathbf{L}^{p}\right) \times\right.$ $\left.\left(\mathbf{L}_{-\sigma}^{2} \cap \mathbf{L}^{2} \cap \mathbf{L}^{p}\right)\right)$ from the above argument for $p \geq 6$. By uniqueness and the continuous 
embedding of $\mathbf{H}^{1}$ in $\mathbf{L}_{-\sigma}^{2} \cap \mathbf{L}^{2} \cap \mathbf{L}^{p}$, we infer that the two solutions must coincide. Therefore, the time decaying estimates also hold for the $\mathbf{H}^{1} \times \mathbf{H}^{1}$ solutions. This completes the proof of Theorem 1.1.

\section{Competing interests}

The authors declare that they have no competing interests.

Authors? contributions

All authors contributed equally to the writing of this paper. All authors read and approved the final manuscript.

\section{Author details}

'School of Mathematics and Information Science, Henan University of Economics and Law, Zhengzhou, 450000, P.R. China. ${ }^{2}$ School of Mathematics and Information Science, Henan Economics and Technical School, Zhengzhou, 450000, P.R. China. ${ }^{3}$ College of Information Technology, Jilin Agricultural University, Changchun, 130018, P.R. China. ${ }^{4}$ College of Mathematics, Jilin University, Changchun, 130012, P.R. China.

\section{Acknowledgements}

The authors express their sincere thanks to the anonymous referees for the comments.

Received: 21 October 2014 Accepted: 8 December 2014 Published online: 10 January 2015

\section{References}

1. Pitaevskii, L, Stingari, S: Bose-Einstein Condensation. Oxford University Press, Oxford (2003)

2. Ambrosetti, A, Colorado, E: Standing waves of some coupled nonlinear Schrödinger equations. J. Lond. Math. Soc. 75, 67 (2007)

3. Belmonte-Beitia, VM, Pérez-García, J, Torres, PJ: Solitary waves for linearly coupled nonlinear Schrödinger equations with inhomogeneous coefficients. J. Nonlinear Sci. 19, 437 (2009)

4. Hioe, FT, Carroll, CE: Coupled Gross-Pitaevskii equations with a square-well and a Coulomb potential. Phys. Lett. A 299, 189 (2002)

5. Roskes, GJ: Some nonlinear multiphase interactions. Stud. Appl. Math. 55, 231 (1976)

6. Yang, J: Multiple permanent-wave trains in nonlinear systems. Stud. Appl. Math. 100, 127 (1998)

7. Nirenberg, L: Topics in Nonlinear Functional Analysis. Chapter 6 by E Zehnder. Notes by RA Artino. Revised reprint of the 1974 original. Courant Lecture Notes in Mathematics, vol. 6. New York University, Courant Institute of Mathematical Sciences, New York (2001)

8. Kirr, E, Zarnescu, A: On the asymptotic stability of bound states in 2D cubic Schrödinger equation. Commun. Math. Phys. 272, 443 (2007)

9. Pillet, CA, Wayne, CE: Invariant manifolds for a class of dispersive, Hamiltonian partial differential equations. J. Differ. Equ. 141, 310 (1997)

10. Cazenave, T: Semilinear Schrödinger Equations. Courant Lecture Notes in Mathematics, vol. 10. New York University, Courant Institute of Mathematical Sciences, New York (2003)

\section{Submit your manuscript to a SpringerOpen ${ }^{\circ}$ journal and benefit from:}

- Convenient online submission

Rigorous peer review

- Immediate publication on acceptance

- Open access: articles freely available online

- High visibility within the field

- Retaining the copyright to your article 